\title{
Access to Facility-Based Childbirth: Women's Experience on Enablers and Obstacles to the Utilisation of Waiting Mothers' Shelters in Rural Zimbabwe
}

Brenda Muchabveyo ( $\square$ muchabveyobrenda@gmail.com )

University of Zimbabwe

\section{Research Article}

Keywords: Waiting Mothers' Shelter, Expectant Mothers, Utilisation, Skilled Birth Attendants

Posted Date: September 24th, 2021

DOl: https://doi.org/10.21203/rs.3.rs-923265/v1

License: (9) This work is licensed under a Creative Commons Attribution 4.0 International License. Read Full License 


\section{Abstract}

This article explores experiences and perceptions of women concerning the utilising a waiting mothers' shelter at Bonda Mission Hospital in the Manicaland province of Zimbabwe. It draws on a phenomenological qualitative research design. This incorporated in-depth interviews and key informant interviews with purposively selected fifteen women who have used the waiting mothers' shelter since 2015 and eight healthcare practitioners respectively. The paper is guided by Alfred Schutz's (1972) social phenomenology. While the findings reveal that most women acknowledged the importance of waiting mothers' shelters in improving access to skilled birth attendance and maternal health outcomes, there are still factors that militate the use of such innovations. Several socio-cultural and economic factors such as constrained decision making among women, mistreatment and lack of privacy in the shelters are some of the deterrent factors. The article concludes that, although waiting mothers' shelters are facilities proven to be beneficial in rural communities, they continually face the risk of not being used. There is a need for a multi-stakeholder approach to address the barriers that deter women from utilising the waiting mothers' shelters and improve access to facility-based delivery, access to skilled birth attendants and enhance the maternal health outcomes in rural communities in Zimbabwe.

\section{Introduction}

Notwithstanding efforts to improve prenatal and postpartum health care in sub-Saharan Africa, maternal healthcare remains an on-going concern. Every expectant mother anticipates a successful delivery of their newborn, nonetheless, maternal mortality remains a highly likely inescapable danger in most rural communities in Africa (Dodzo and Mhloyi 2017). In many cases, pregnancies end in fatalities of either mother or the newborn or both. One of the primary reasons for this are non-hospitalised deliveries without skilled care, in places without appropriate or adequate facilities to handle complications and emergencies should they arise (Mafaune et al., 2020). Several notable factors lead to non-hospitalised deliveries in rural communities. These include physical and logistical obstacles such as long distances to healthcare facilities, high transportation costs, poor quality of services due to low staffing levels and lack of medical equipment for emergence obstetric care among others (Kanengoni et al., 2019). As a result of high maternal deaths due to non-hospitalised deliveries, numerous measures have been initiated to mitigate maternal and perinatal mortality in rural communities where healthcare facilities are not proximate. One such measure is the establishment of facilities called waiting mothers' shelters.

Waiting mothers' shelters have been conceptualised as "housing facilities" near a qualified medical facility (hospitals). In waiting mothers' shelters, expectant mothers can await their delivery and be transferred to a nearby medical facility shortly before delivery, or earlier should complications arise (World Health Organisation [WHO] 1996a: 1, Yustina 2020). Expectant mothers do not give birth at the waiting mothers' shelter, but rather await delivery there in the weeks before they are due to give birth. They are transferred to the health facility when they go into labour (the stage when one is confirmed by the attending healthcare workers to be in labour). The waiting mothers' shelter strategy in developing countries' maternal health systems aims to reduce maternal and infant mortality by decreasing the 
physical distance that precludes rural expectant mothers from receiving unsurpassed maternal healthcare and emergency obstetric care (WHO 1996a, Yustina 2020).

The establishment of waiting mothers' shelters in African countries has been advantageous because it emphasises the importance of giving birth in a hospital under the supervision of qualified skilled birth attendants with emergency facilities should any complications arise (Slalubanje et al., 2014).

Additionally, waiting mothers' shelters are beneficial in most developing counties across sub-Saharan Africa (Mafaune et al., 2020). In countries such as Ethiopia, Liberia and Eritrea, waiting mothers' shelters have improved expectant mothers' access to healthcare facilities, lead to an increase in the number of expectant mothers receiving skilled birth attendance, reduced maternal mortality and improved maternal and new born health outcomes among women who stayed there during the last period of their pregnancy (Slalubanje et al., 2014). Moreover, waiting mothers' shelters have been reported to be essential in the management of women with high-risk pregnancies[1] (Mafaune et al., 2020). The waiting mothers' shelters have proven to be facilities beneficial to expectant mothers in rural communities who lives far away from health care facilities and those with risk factors.

Zimbabwe currently has 1335 health facilities, with a waiting mothers' shelter available in or close to 255 of these facilities (Zimbabwe National Statistic Agency [ZIMSTAT] 2012: 82). Additional shelters are being built near rural hospitals following the government mandate to establish a shelter at every hospital in rural Zimbabwe (under The Zimbabwe National Maternal and Neonatal Health Road Map Policy of 2007-2015 introduced by the Ministry of Health and Child Care [MoHCC]). According to MoHCC (2016: 59), one in six referral hospitals has a waiting mothers' shelter. As part of the implementation of the Zimbabwe National Maternal and Neonatal Health Road Map Policy of 2007-2015, the government of Zimbabwe took action concerning waiting mothers' shelters to make them effusively functional and ensure that all expectant mothers living far away from health care facilities are in contact with health facilities for prenatal, intra-, and postpartum care (MoHCC, 2016). The government of Zimbabwe recruited registered nurses nationally to be trained in midwifery and strengthened the capacity of the midwifery training school to have competency-based midwifery training (MoHCC, 2016). Availability of five midwifery tutors for each school was ensured whilst policies for retention of midwives were strengthened. For example, "bonding midwife nurses to an equivalent of several years of training received so that they would at least serve at waiting mothers' shelters as part of their training was introduced (MoHCC 2016: 200)."

However, as much as the government has made efforts to make the waiting mothers' shelters fully functional in rural Zimbabwe, low utilisation of these facilities has been reported and only $31 \%$ of the expectant mothers in rural areas use these facilities (Musuka et al., 2021). This is due to various factors including the cost associated with staying in this facility, hardship of staying away from home and the absence of health personnel in healthcare facilities (Mafaune et al., 2020). Diminutive research on women's perceptions towards waiting mothers' shelters has been conducted in Zimbabwe. Research findings from other countries may not apply to the rural Zimbabwean context. Women's experiences and opinions regarding utilisation of the waiting mothers' shelters may also differ from one geographical, 
socio-cultural and economic context to another. Thus, the purpose of this paper is to explore experiences and perceptions of women concerning the enablers and barriers to the utilisation of waiting mothers' shelter in rural Zimbabwe.

This article is influenced by Alfred Schutz's (1972) social phenomenology. This framework studies the phenomenon based on what people experience in their daily life, considering them as elements that act, interact and understand one another within the social world. Schutz (1972) emphasises ways in which actors in the social world construct reality. Social phenomenology aims to understand the social world in terms of inter-subjective meaning. In the sociology of medicine, issues to do with health and illnesses in the society also draw upon social phenomenology through examining how the health of individuals, larger social formations, and the environment are affected by inter-relationships between humans and the social construction of reality (Zaman 2005). Ferzacca (2005) and Geest (2003) also emphasise on a meaning-centred perspective in unravelling the phenomenological meanings of healthcare systems in society and how individuals experience them. Thus, it is essential to use this framework to understand the experiences of expectant mothers in the waiting mothers' shelters regarding sociocultural factors that influence their use or non-use of the shelters in rural Zimbabwe.

[1] According to Lonkhuijzen et al., (2012), the concept of risk selection aims to select expectant mothers for referral to a waiting mothers' shelter. Selection takes place during antenatal clinics by the attending health professional, either within the hospital or in health centres/clinics without labour facilities. Selecting women with risk factors such as a poor obstetric history, high parity or anaemia and advising them to stay near a hospital could prevent maternal or infant mortality during or immediately after delivery.

\section{Methodology}

In order to address the goal of this paper, a phenomenological qualitative design which incorporated indepth interviews (IDIs) and key informant interviews (KIIs) were used. This provided a detailed understanding of the women's experiences and perceptions concerning the enablers and obstacles on the utilisation of waiting mothers' shelters in rural Zimbabwe. Women who had used the waiting mothers' shelter since 2015 were engaged through (IDIs) as the healthcare professionals were also engaged through (KIIs).

\section{Sampling}

The central unit of analysis for this article was women who have used the waiting mothers' shelter at Bonda Mission Hospital since 2015. This time frame is significant due to the government mandate that all hospitals in rural Zimbabwe should have a waiting mothers' shelter under the Maternal and Neo Natal Health Road map 2007-2015 policy (MoHCC 2016, United Nations Population Fund [UNPF] 2016). Expectant mothers who participated in this research were from different age groups (18-45) and geographical areas regardless of class and marital status in Mawadza village. The age groups 18-45 
were dominant because 18 years is the legal age for consent in Zimbabwe and 45 was the furthest that participants consented to participate in the study.

In addition, KIls in this study were doctors, nurses and midwives. Two representatives were chosen from each category of KIls but midwives had four representatives to capture the narratives of those trained by the (MoHCC) and those who had gone through the training of Zimbabwe Traditional Healer's Association [ZINATHA]. Two representatives provided broader experiences from those on the ground and those involved in supervisory tasks.

\section{Data Collection Procedure}

The data collection process in this study was from between June and November 2019 in Mawadza village in Mutasa District in Manicaland, Zimbabwe. Mawadza village falls under Mutasa Rural District Council [MRDC] in Manicaland province. The province is estimated to have a population of $1,755,000$ according to the (ZIMSTAT 2012: 213). Manicaland Province is subdivided into six districts, namely, Mutare, Chipinge, Chimanimani, Buhera, Makoni and Mutasa. Mutasa district is located thirty kilometres from, Mutare which is the provincial capital city (Musuka et al., 2021).

Bonda Mission Hospital is a mission hospital run by the Anglican Church. This hospital is the largest in Mutasa district with a threshold that goes beyond Mutasa district. In addition, Bonda Mission Hospital remains the only option for villagers in Mawadza, which is the case study area for this research. The hospital is an Anglican Mission hospital that has an associated school of Nursing and Midwifery. Bonda Mission has 50 nurses and ten doctors (Musuka et al., 2021). The waiting mothers' shelter is located within the hospital premises and there are houses where expectant mothers await delivery. As part of the amenities at the shelter, there are two houses, Blair toilets and water tanks to ensure access to clean water. One of the houses accommodates those who are awaiting labour and the other accommodates those receiving postpartum care after childbirth.

A total of 23 interviews (IDIS and KIIs) were conducted which lasted for approximately 60- 80 minutes each. The interviews were conducted in the Manyika dialect, the local language used by the people in the Mawadza community. The data collected from the interviews were audio-recorded by the author and then transcribed and translated to English afterward. The author is Zimbabwean by birth, descent and she is female. She speaks Shona, particularly ChiManyika dialect, the predominant variety used by people in Mawadza community. This study was about women and was carried out by a woman to implement what Oakley $(1975 ; 1980 ; 1988)$ advocated for (study of women by other women) to eradicate gender biases in sociological childbirth studies. The author also resides in Mutare, the Manicaland provincial capital, about 50 kilometres from the case study research area. She has a strong command and knowledge of culture and childbirth issues in the case study area.

\section{Data Analysis}


Qualitative data from the IDIs and KIls were entered into an excel matrix. These data were then analysed both deductively and inductively, coding both according to a framework that was based on the IDI and KIls topic guide, but also with an iterative approach to emerging themes. These data were then analysed thematically for patterns, trends, and outliers to explore.

\section{Ethical Considerations}

The study got ethical approval from the MoHCC Manicaland Provincial offices. This research methodology was considered to be of no physical risk and minimal psychological risk to participants and the data was anonymised during the writing process to ensure confidentiality.

\section{Research Findings}

The section analyses the women's experience on enablers and obstacles to utilising waiting mothers' shelters at Bonda Mission Hospital. It looks at the social processes associated with these experiences from the narratives and nuances of the women themselves.

\section{Need to Access Skilled Birth Attendants}

The need to access skilled birth attendants has encouraged expectant mothers to use the waiting mothers' shelter at Bonda Mission Hospital. The necessity to use the waiting mothers' shelter varies with the medical conditions of the expectant mothers, birth experiences and complications, among other factors. Most expectant mothers interviewed agreed that they needed the shelter because they were safe in the hands of healthcare workers who had experience in handling pregnancies. Participant (i) stated that:

I always want to await labour in the shelter because it gives me access to nurses and doctors whenever I need them. Right now, the country is going through strikes, but we have midwives on duty and they constantly teach us how to do the correct thing with our pregnancies. I think that is very important to me as a mother.

In agreement with Participant (i), Participant (ii) stated that:

I am an HIV-positive pregnant mother and my condition requires me to give birth at a hospital to save my new-born child from getting infected. Therefore, I need qualified health care workers to assist me during delivery; so, waiting for delivery in the shelter is important.

Postpartum care received in the shelter has also been a major driving force for women to utilise the waiting mothers' shelter. The fear of post-delivery haemorrhage, which usually results in the death of new mothers in this area, has driven mothers to use the shelter for proper assessment before they are discharged out of the facility.

$\mathrm{KI} 1$, who is a doctor, had this to say: 
Up to $98 \%$ of the mothers who use the shelter do not suffer from post-delivery haemorrhage, and the last recorded case in this hospital was handled well with proper medication to stop the new mother's profuse bleeding. Other common conditions are Yellow fever and Jaundice among newborn babies. This requires extra check-ups and care and when mothers experience this, they realise they need to await labour in the shelter in order to receive comprehensive postpartum care.

Narratives presented by the participants show that there is a growing dependency on the waiting mothers' shelter among facility users due to the services offered and this has been supported by some of the bylaws in the community that support the use of the mothers' shelter. The existence of these by-laws has also facilitated the increased utilisation of the waiting mothers' shelter.

\section{Criminalisation of Home Births in Surrounding Villages}

The communities, through by-laws, have criminalised homebirths.Chiefs and village heads penalise families who give birth at home. All participants acknowledged that when one gives birth at home, the family pays a fee to the chief they can obtain a letter to the hospital acknowledging a new birth in his village book. This letter serves as proof of delivery so that the newborn can be put on an immunisation plan and, later on, be eligible for registration in the registry department for issuance of a birth certificate. Participants noted that without the letter from the chief, one could not register one's newborn baby; hence, the baby will not have a birth certificate or national identity or any other documentation.

$\mathrm{KI} 2$ further remarked that:

The MoHCC has not totally disqualified but does not categorically encourage home births, especially in this Zimbabwean context, and mostly in our rural communities. Community by-laws then act as a social control mechanism to support the use of formal maternal health care facilities by rural women so as to reduce maternal mortality, especially during or soon after delivery. These by-laws have been useful in encouraging expectant mothers to attend prenatal care at the hospital and come to the waiting mothers' shelter to await delivery.

Although the aforementioned factors have been push factors for women to use the waiting mothers' shelter, participants noted other challenges that still deter them from using the waiting mothers' shelter at Bonda Mission Hospital.

\section{Inadequate Knowledge about the Waiting Mothers' Shelter}

Inadequate knowledge about the waiting mothers' shelter is one of the reasons why some women choose not to await labour in the waiting mothers' shelter. The inadequate knowledge is also gendered as men are reported not to know its operations, usefulness, and benefits of using the waiting mothers' shelter in the prenatal and postpartum stages. Expectant mothers know of the benefits of awaiting labour at the shelter through their experience in this facility. There is not enough awareness about the actual activities that occur in this facility. Participant (iii) articulated that: 
I only had to know this when I came here. Back in our villages we think coming to the shelter is for weak expectant mothers who want to be relaxing for two weeks before giving birth. I think we used to think so because we did not get enough information about the shelters.

Inadequate knowledge of the activities that occur in the shelter and the benefits that women have by awaiting labour is a deterrence to the utilisation of this shelter. In addition, the fact that men also have inadequate knowledge of the shelter especially its purpose and functions has further deterred women from using the shelter since they lack ultimate decision making power to leave their houses for some time as they await labour.

\section{Matrix 1: Enablers and Obstacles to the Utilisation of Waiting Mothers' Shelters}

\section{Enablers}

- Criminalisation of Home Births

- Need to access skilled health workers

- Need to prevent complications

\section{Obstacles}

- Women's low decision-making power to use the shelter.

- Domestic expectations and Gender Roles

- Lack of privacy in the shelter

- Economic challenges

- Long Distances to the Shelter and Lack of Effective Transportation System

\section{Women's Lack of Decision-Making Power to Use the Shelter}

The participants depend on their husbands' authority to use the shelter. For those who do not have husbands, a male member of the family's authority is needed before going to the shelter. Expectant mothers indicated their interest to await labour in the shelter but they are sometimes not allowed by their husbands. Some participants noted that they awaited labour in the shelter for their first pregnancies only but were not allowed by their husbands to do the same for the second time and some for the third time even. Participant (iv) narrated that:

My husband did not want me to keep on going to the shelter after my first pregnancy. He kept on explaining to me that there was no need to spend resources using the shelter every time I fall pregnant as it was going to be difficult for him to fund for that.

Key informants noted that most women come to the shelter with their husbands' authority because they do not have the decision-making power to come to the shelter without the husband's consent. This is because husbands usually face challenges in taking care of the families when their wives are away.

\section{Family Expectations and Gender Roles}

Expectant mothers also highlighted that they have family commitments that require their ongoing presence at their homesteads. In this culture, women (mothers) are responsible for a number of tasks 
such as household chores and child-rearing, apart from child bearing. The fathers usually do not want to let go of their wives to go to the shelter for they do not want to be burdened by household chores and child rearing duties. Most men prefer that the expectant mother's pregnancy be monitored at home in order to avoid house hold chores since they will be committed with manual labour to provide for their families. Participant (v) noted that:

I have so many commitments at home that require my attention as a mother. I have other young children who still need my care. I ended up taking them to my mother for proper care when I am away. I also have a field that is not being tilled at the moment because the people who assist me cannot come when I am not there. Coming to the shelter disrupts the normal way of life but there is no option because we need to save the babies' lives and our lives too.

Family commitments are a notable reason why women are not utilising the waiting mothers' shelter. In Mawadza community gender roles according to the societal beliefs, women are expected and required to be in the private sphere (the home). The involvement of women in household chores is influenced by the societal culture. Gender roles have gone to the extent of influencing the childbirth process in Mawadza rural community.

\section{The Increasing Involvement of Men in the Hospital Childbirth Process}

The childbirth process at Bonda Mission Hospital increasingly involves men. Contrary to that, the birth process in this area has traditionally been handled by women (female midwives) both in hospital deliveries and home births. Over the years, there have been a considerable number of men joining the field of gynaecology, obstetrics and generally being trained to be midwives at Bonda Mission Hospital. The increase in the number of men joining this field has also gone along with the fluidity of most gender roles in contemporary societies. In maternal health, specifically, in Zimbabwe, this has been influenced by the growing medicalisation of the birth process and the need for more skilled birth attendants; hence men end up joining, if not dominating the sphere. Participant (vi) noted that:

I am not comfortable with male midwives in the shelter; they make me so shy, and it's against our culture to expose our bodies to males - regardless of the help they could be offering. The males are even rude. They keep on telling us to be professional because they would be at work and not there for jokes.

The increasing involvement of men in hospitalised birth is an area of major concern from the narratives of a significant population of the participants. A closer analysis shows that the concerns of the women arise from their cultural beliefs and the respect of the women's reproductive system and genitals more specifically in this culture. The dismantling of this tradition results in women not being comfortable giving birth in the hospital and resorting to the perceived dignified home birth surrounded by women.

\section{The Relationship between Expectant Mothers and Health Care Workers in the Shelter}

Overall, participants highlighted many reasons influencing their decision to either use or not use the waiting mothers' shelter to await delivery. Expectant mothers indicated that healthcare workers often 
mistreat or demean them during their antenatal visits and their stay in the waiting mothers' shelter. Additionally, health workers agreed that expectant mothers often do not receive the care they deserve due to staff shortages owing to the current economic hardships. None of the TBAs interviewed listed the relationship between expectant mothers and health care providers as the reason why expectant mothers do not await delivery in the waiting mothers' shelter. It had been a barrier for most expectant mothers, and a reason why they think their peers do not use the waiting mothers' shelter. Participant (vii) noted:

The treatment we get in this shelter is harsh at times. The midwives usually shout at us and they do not care how we feel after they shout at us. I would like to see in this shelter that, when an expectant mother comes into the shelter, she should be treated with respect and dignity like any other human being. They should treat us like their daughters, not shout at and beat us ... So, I would love to see the midwives welcoming and treating us well.

Expectant mothers stressed that their mistreatment by the health care providers amounted to what they believed was a misuse of their authority. Expectant mothers stated that they felt helpless in these situations as they rely on these professionals for care. Furthermore, since most of the expectant/new mothers were of lower socio-economic status, they feared speaking up or were unsure what to say in those situations.

Health care workers admitted that it was often difficult to maintain positive attitudes throughout the day due to staff shortages, with the current economic hardships being faced in Zimbabwe. The health care professionals noted that the patient load is often greatest at these facilities than at the primary and secondary levels. In addition to staff shortages, health workers confirmed that expectant mothers were mistreated or shouted at when they presented late for care or did not follow simple instructions. $\mathrm{KI} 4$ stated that:

I think much of it is the personal attitude; it could also be related to the long shift we are taking these days. At the moment, we are not short-staffed in theory but in practice, people show up for work for a few hours and then disappear because nurses and doctors are on strike nationwide. You may find that one doctor could be having one shift that he is doing; he has one shift and another shift and another shift, and they are tired. (Furthermore), you are telling this expectant mother (what is wrong), and she does not understand you, and the expectant mother is doing something else and then you yell on top of your voice.

The participants have noted that the situation in the shelter has been worsened by a lack of respect and lack of privacy in this facility. This is an area that they possibly would like to see improve.

\section{Economic Challenges, Long Distances and Lack of Proper Transport System}

Furthermore, among the reasons women do not use the waiting mothers' shelter up to the level it is supposed to be used, are the long distances that exist between the expectant mothers' homes and Bonda Mission Hospital, lack of proper transportation to the health care facility and a poor road network. Participants indicated that they use donkeys, bicycles and motorbikes as transport to the 
hospital for prenatal care before they are due for their waiting period in the shelter. This has posed challenges to them because it is difficult to use a bicycle until one reaches the hospital with the state of the road network. Hiring a car or a motorbike is expensive for most expectant mothers. Participant (viii) stated that:

I was given a bicycle to use by my husband, but, it's not efficient because the terrain is not flat before I reach the hospital using the shortcut.

Economic challenges have worsened the long distances. When the expectant mother is due to go to the shelter, she is required to bring provisions such as a notebook, firewood, food, pots, blankets, buckets, toiletries, including detergents to clean the toilets, latex gloves, cotton wool, candles, razor blades, shaving cream, and presentable clothing - preferably nightwear. These provisions are usually required to be utilised by the expectant mothers during their stay in the shelter.

Due to economic challenges expectant mothers fail to provide all these requirements to be eligible to await labour in the waiting mothers' shelter. Participant (viiii) articulated that:

It is very hard in this economy to have all the provisions needed to stay in the shelter. At the end of the day, when you realise you do not have enough, you will just resort to giving birth at home

The challenges of providing one's consumables while awaiting labour in a waiting mothers' shelter are common in Mawadza village. Complications of pregnancy and childbirth can pose severe risks to the health of expectant mothers, especially in resource-poor rural settings. These result in some not meeting the required standards, so they resort to non-use of the facilities. Intervention from governments and other stakeholders to assist with provisions such as food and sanitary wear is vital to encourage those without access to these also to use the waiting mothers' shelters.

\section{Discussion Of Findings}

This paper aimed to explore experiences and perceptions of women concerning utilisation of a waiting mothers' shelter at Bonda Mission Hospital in Manicaland, Zimbabwe. The results reveal that experiences that expectant mothers go through in their everyday life are influential in constructing their reality and ultimately work as enablers or obstacles to the use or non-use of the waiting mothers' shelter. Phenomenology has, thus, provided a framework to attend to various aspects of women's lived experiences in Mawadza rural community by providing a platform for discerning and describing human experience and understanding the particular background against which action is perceived and interpreted (Maron, 2004).

There are different aspects of how women's childbirth experiences through interaction are shaped by society and this ultimately influences women's choice of birth territory (Fielder 1996). Firstly, indigenous knowledge and cultural factors in this particular society play a pivotal role in shaping women's experiences which influence their choices of use or non-use of the waiting mothers' shelter. Indigenous 
knowledge and cultural factors in this case point to social stratification according to gender that influences the knowledge social actors to hold about the waiting mothers' shelter including its function and purpose. In Mawadza rural community, men's and women's experiences are different due to gender stratification as women are better informed about the shelter as compared to men. In their experiences, which ultimately shape their lived reality men do not see the importance of utilising the waiting mothers' shelter whereas women do. In this patriarchal society where men are the ultimate decision-makers women face a risk of not being supported by spouses or other male members of the family to use the shelter due to the inadequate knowledge men have about the waiting mothers' shelters.

Apart from, stratification according to gender, family commitments and gender roles have also played a role in defining the experiences and lived realities of expectant mothers in Mawadza rural community. Through socialisation, men and women are taught different roles according to the culturally accepted norms and values of any given society. As such, women are entitled to childbearing, rearing and other household chores as men are into manual labour to fend for the families in Mawadza community. The allocation of gender roles through socialisation has shaped women's experiences as they internalise these roles as part of their cultural beliefs and identity. The internalisation of gender roles has affected the use of birth territories in this community because roles such as childrearing and other household chores entail that a woman should be at the home to ensure that these roles are fulfilled effectively. Literature has also confirmed the findings of this study by showing that the waiting mothers' shelters were introduced in most parts of rural Ghana; however, Ahafo Ano and Adansi district failed to sustain them (Lohela et al., 2012). This was due to bias towards home delivery by most members in indigenous communities in Ghana (Walraven et al., 2000). In addition, women could not take care of their families and their farms when they were in the shelters. In Nicaragua, being away from the family was also considered the main drawback of using a waiting mothers' shelter (Lohela et al., 2012). Gender roles and family commitments have been an obstacle towards the utilisation of the waiting mothers' shelter even in other regions across sub-Saharan Africa.

The fluidity of gender roles has also shaped the lived experiences of people in society today, where previously male-dominated sectors are being filled by women and vice versa. The increasing involvement of men in the hospitalised childbirth process has worked as a major obstacle towards utilising the waiting mothers' shelter. This is mainly due to the cultural beliefs in this indigenous community that childbirth is a process that should be handled by women hence men are generally not allowed in traditional birth territories. Community members have not accepted the shifting gender roles since they come in antagonism with the culturally accepted gender roles in Mawadza community hence it has deterred most expectant mothers to utilise the waiting mothers' shelter.

A phenomenological analysis of health and illness issues in sociology points to the importance of social relations and social networks and environment in shaping the lived experiences of social actors (Schutz, 1972). The nature of one's social network affects the person's lived experience and this ultimately shapes one's lived reality. The community through by-laws have influenced the lived reality of expectant mothers by discouraging home births; hence families and expectant mothers go to the waiting mothers' shelter to 
await delivery. In this context, healthcare-seeking behaviours are influenced by the social networks Zaman (2005) and Geest (2003) of the expectant mothers interact with and how the network shapes their lived realities. If an expectant mother's social network is highly inclined towards using biomedical health care for childbirth, she is likely to go to the waiting mothers' shelter to await delivery and give birth at the nearby Bonda Mission Hospital. Those whose social networks are skewed towards traditional ways of childbirth are likely to give birth at home although communities are criminalising homebirths. In some ways, the size of the social network affects the breadth and depth of the experiences one can draw from in interpreting the world (Maron, 2004).

Phenomenology has been useful in understanding the overall experiences patients have of their healthcare system (Little, 2001). In this case, it has been useful in understanding the experiences of expectant mothers in the waiting mothers' shelter at Bonda Mission Hospital. Much has been written on patient-centred care (Fulford 1996; Little 2001) but overall patient complaints about particular aspects of their care remain common. In addition, there is no generally accepted and methodologically robust way of measuring patient satisfaction (Sitzia \& Wood, 1997). By understanding through a phenomenological lens the experience of interacting with healthcare professionals in a clinical setting, a better understanding of these experiences has been achieved and improvements could be made. Expectant mothers have indicated complaints about their relationships with their health care providers.

The lived experiences of expectant mothers in the shelter have been summed up by the majority of the participants as being filled with abuse from healthcare providers and this has worked as an obstacle to the future use of the shelter as the majority of the participants fear being abused by the healthcare providers. In as much as waiting mothers' shelters have proved to be beneficial facilities, a study from Ghana by Wilson et al., (1997) showed low utilisation of the waiting mothers' shelter due to various factors including the cost associated with staying in a waiting mothers' shelter. In southern Malawi not all hospitals have a shelter hence women lodge in wards (Lohela et al., 2012). There is no supervision by midwives and poor staff attitudes during antenatal care and delivery. In the DRC there is low use of shelters due to lack of privacy, inability to use traditional birthing practices, lack of respect from health staff, and the cost of reaching the hospital (Eckermann 2008). In addition, understanding the experiences of the expectant mothers from their own voices and nuances according to their lived experiences is important for it helps in the improvement of the shelter based on the experiences of those who have utilised it.

Once the experience of illness in the sociology of medicine is better understood using phenomenological tools, this understanding can assist in devising useful interventions based on specific knowledge of what would impact positively on the life-world of the care seekers in any given society. Interventions can then be tailored to target real, rather than assumed, needs and would therefore offer greater benefit to care seekers who are expectant mothers in this context.Interventions may also identify needs beyond the medical condition itself, allowing improved quality of life. Although much work has been invested in this area, the rich understanding that phenomenology has offered can advance existing work.It is important to study the experience of expectant mothers in biomedical healthcare birth territories such as waiting 
mothers' shelters. Thus providing various actors with a concrete understanding of the impact of the experiences of expectant mothers towards the utilisation of these facilities.

\section{Recommendations And Conclusions}

Basing on research findings, it can be recommended that improvements on maternal health related issues in Zimbabwe should emphasise the low social status of women and provide them with adequate information, skills and resources to safeguard decision-making independence with regard to childbirth in any health care facility of their choice. Partner/Spouse involvement should also be promoted by providing husbands with adequate knowledge about the waiting mothers' shelter, their purpose, function and how utilising these facilities would improve facility-based delivery in rural communities in Zimbabwe. Finally, there is need to consider the provision of basic needs such as adequate sleeping space, beddings, water and sanitary services, and food and cooking facilities and an improvement on the caregivers' attitude in the waiting mothers' shelters. These improvements will encourage expectant mothers to utilise the waiting mothers' shelters and have access to skilled birth attendants and improve maternal outcomes.

In conclusion, this paper has established experiences and perceptions of women on the factors that enable or deter them from utilising the waiting mothers' shelter at Bonda Mission Hospital. It has been shown that waiting mothers' shelters are beneficial maternal healthcare facilities which improve access to and utilisation of facility-based skilled birth attendance services. The participants have shown that the waiting mothers' shelter is being utilised for two main reasons; that is the need to access skilled birth attendance and community by-laws that support the criminalisation of home births in this area. This process has encouraged women to await labour in the waiting mothers' shelter so that they can give birth in the hospital where there are skilled birth attendants. However, the benefits of awaiting labour in the waiting mothers' shelter are not fully realised due to various factors. Some of the factors include sociocultural issues, inhibited decision making among women among other factors. There is need for a multi-stakeholder approach to address the barriers that deter women from utilising the waiting mothers' shelters and improve access to facility cased delivery and access to skilled birth attendants in order to improve the maternal health outcomes in rural communities in Zimbabwe

\section{References}

1. Archer, M.S. 1995. Realist Social Theory: The Morphogenetic Approach. Cambridge University Press.

2. Dodzo, M.K., and Mhloyi, M., 2017. Home is best: Why Women in Rural Zimbabwe Deliver in the Community. PLoS One, 12(8).

3. Eckermann 2008. Eckermann E, Deodato G. Maternity Waiting Homes in Southern Lao PDR: the unique 'silk home'. Journal of Obstetrics \& Gynaecology Research, 34(5):767-75.(PubMed: 18834334). 
4. Ferzacca, S., 2005. Mediations of Health and the Development of a Nation: Late Suharto, Late Modernity. In Liberalizing, Feminising and Popularising Health Communications in Asia. Routledge.29-56.

5. Fiedler, D.C., 1996. Authoritative Knowledge and Birth Territories in Contemporary Japan. Medical Anthropology Quarterly, 10(2):195-212.

6. Fulford, K.W. M et al. (1996). Essential Practice in Patient-Centred Care. London: Blackwell.

7. Geest, S. and K. Finkler. 2004. Hospital Ethnography: Introduction. Social Science and Medicine, 59(10):1995-2001.

8. Kanengoni, B., Andajani-Sutjahjo, S., and Holroyd, E., 2019. Women's Experiences of Disrespectful and Abusive Maternal Health Care in a Low Resource Rural Setting in Eastern Zimbabwe. Midwifery, 76: 125-131.

9. Little P., H. Everitt, I. Williamson, G. Warner, M. Moore, C. Gould, K. Ferrier \& S. Payne (2001). Preferences of Patients for Patient Centred Approach to Consultation in Primary Care: Observational Study. British Medical Journal, 322(7284):468, doi:10.1136/bmj.322.7284.468.

10. Lohela, T.J., Campbell, O.M., and Gabrysch, S., 2012. Distance to care, facility delivery and early neonatal mortality in Malawi and Zambia. PloS one, 7(12), p.52.

11. Mafaune, H.W., Sacks, E., Chadambuka, A., Musarandega, R., Tachiwenyika, E., Simmonds, F.M., Nyamundaya, T., Cohn, J., Mahomva, A. and Mushavi, A. 2020. Effectiveness of Maternal Transmission Risk Stratification in identification of Infants for HIV Birth Testing: Lessons from Zimbabwe. Journal of Acquired Immune Deficiency Syndromes (1999), 84(1), p.28.

12. MoHCC, An Analysis of Notified Institutional Maternal Deaths, January 2015-December 2016.

13. MoHCC, The National Health Strategy for Zimbabwe (2010-2016), Equity and Quality in Health: A People's Right, MOHCW, 2017.

14. Musuka, G., Dzinamarira, T., Cuadros, D., Chingombe, I., Murewanhema, G. and Mapingure, M., 2021. Access to Health Insurance in Zimbabwe and associated factors: Insights from data from the Zimbabwe Demographic Health survey, 2015.

15. Oakley, A., 1976. Wise woman and Medicine Man: Changes in the Management of Childbirth. The rights and wrongs of women, pp.17-58.

16. Oakley, A., 1980. Women Confined: Toward a Sociology of Childbirth.

17. Oakley, A., 1988. Is Social Support Good for the Health of Mothers and Babies? Journal of Reproductive and Infant Psychology, 6(1): 3-21.

18. Schütz, A., 1972. Der Fremde. In Gesammelte Aufsätze (pp. 53-69). Springer, Dordrecht.

19. Sialubanje C, Massar K, Hamer DH, Ruiter RAC. 2014. Psychosocial and Environmental Predictors of the Intention to Use Maternal Healthcare Services in Kalomo, Zambia. Health Educ Res, 29(6):102840.

20. Sitzia J. \& N. Wood (1997). Patient Satisfaction: a Review of Issues and Concepts. Social Science and Medicine, 45(12):1829-1843. 
21. United Nations Population Fund (UNFP) .2016. Guidelines for Monitoring the Availability and Use of Obstetric Services. New York: United Nations Children's Fund.

22. Van Lonkhuijzen, L., Stekelenburg, J. and van Roosmalen, J., 2012. Maternity Waiting Facilities for Improving Maternal and Neonatal Outcomes in Low-Resource Countries. Cochrane Database Syst Rev, p3-10.

23. van Lonkhuijzen, L., Stekelenburg, J. and van Roosmalen, J., 2012. Maternity Waiting Facilities for Improving Maternal and Neonatal Outcome in Low-Resource Countries. Cochrane Database of Systematic Reviews, (pp8-10).

24. Wilson, R., 1997. Human Rights, Culture and Context: Anthropological Perspectives.

25. World Health Forum. 1996; 11(4):440-6.

26. Yustina, I., 2020. The Relationship of Maternal Health Conditions on the Utilisation of Maternity Waiting Homes for Maternity Motherhood. Britain International of Exact Sciences (BIoEx) Journal, 2(1): 220-230.

27. Zimbabwe National Statistics Agency (ZIMSTAT), Quarterly Digest of Statistics 2013, ZIMSTAT, 2013.

\section{Figures}




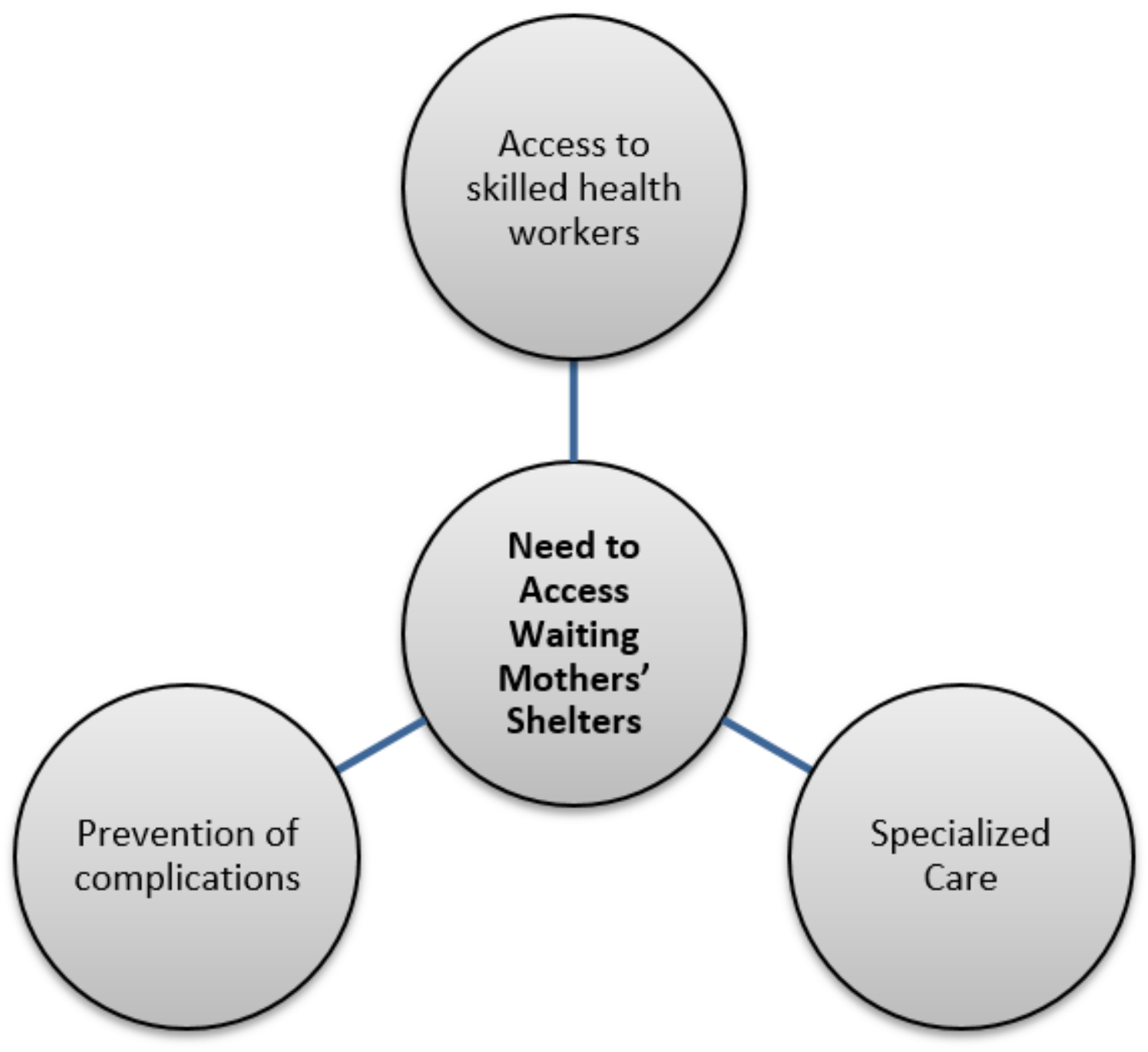

Figure 1

Factors for Perceived Need to Access Waiting Mothers' Shelters 